\title{
PROBLEMGESCHICHTLICHE NOTIZEN ZUR „ARCHITEKTONIK" ALS „PRINZIP DER SUCHE“
}

FABIAN ERHARDT

\begin{abstract}
In the following considerations we want to demonstrate that "architectonics" in the Kantian sense, as the "zetetic principle of search", forms an informative tool to address the dynamization of the transcendental, an extremely productive motif of contemporary philosophy in general, and of phenomenology in particular. Furthermore, we want to outline the extent to which Marc Richir tries to make the concept of architectonics fruitful in order to address the "phenomenality of phenomena" in its irreducible contingency, without concealing it in an archaeological foundation or teleological finalization.
\end{abstract}

\section{Einleitung}

Kants Begriff der Architektonik spielt in Marc Richirs „philosophische[r] Reflexion der Phänomenalität der Phänomene nur als Phänomene "1 eine zentrale methodologische Rolle. Um den metaphysischen, empirischen, logischen, psychologischen, eidetischen oder existenzialen „Fallstricken“ zu entgehen, die alle darauf hinauslaufen, die gesuchte Phänomenalität auf eine jeweilige Weise „zu vergegenständlichen “ und damit zu verfehlen, sind „endlos variab[le] und labil[e] Begriffe" 2 erforderlich - die Architektonik bildet den Inbegriff ihres stets nur provisiorisch konkretisierbaren Zusammenhängens. Denn jede Teleologisierung,

1 Richir Marc, Phänomenologische Mediationen. Zur Phänomenologie des Sprachlichen, Aus dem Französischen von Jürgen Trinks, Wien, Turia + Kant, 2001, S. 9.

2 Ibid. S. 9.

https://doi.org/10.14712/24646504.2020.18

(C) 2020 The Author. This is an open-access article distributed under the terms of the Creative Commons Attribution License (http://creativecommons.org/licenses/by/4.0). 
jede Letztbegründung des Zu-Denkenden gibt Richir zufolge die „Sache selbst“ der Phänomenologie preis: „,[D]ie so unaufhörliche wie rätselhafte Erzeugung und Bildung des »Sinns«" 3 .

Besonders bemerkenswert ist hierbei, dass Kant und Richir trotz aller Differenzen ihres jeweiligen Verständnisses von Architektonik eine fundamentale Perspektive teilen, die ich als das zetetische Prinzip architektonischer Erkenntnis bezeichnen möchte. Auch wenn sich die transzendentale Aufgabenstellung von der Selbsterkenntnis der Vernunft hin zu einer Selbsterfahrung des Sich-bildenden-Sinns verschiebt, meldet sich Architektonik bei beiden Autoren als eine weder logisch noch ontologisch gegründete „Form von Koordinierungsfunktion von Grundlagen“4, die „einen ganz spezifischen Begriff von Einheit als Verständlichkeitsbedingung "5 ermöglichen soll - einer Einheit, die sich Richir zufolge nur „in der unendlichen Bewegung der Suche nach ihr" ${ }^{\text {"6 }}$ realisieren kann. Als ideengeschichtlicher Kontext soll uns zu anfangs eine knappe Skizze der Kontingenz sub specie modernitatis dienen.

\section{Neuzeit: Kontingenzdämmerung}

Kontingent ist das Nicht-Notwendige, also alles, was auch hätte nicht sein können oder auch hätte anders sein können. „Kontingenz“ bezeichnet damit nicht Unbestimmtheit überhaupt, sondern ,jene spezifische Unbestimmtheit, in der etwas weder notwendig noch unmöglich ist"7. Während sich in der Antike das Kontingente als Zufallsbereich des Handelns darstellt und so „die Ambivalenz

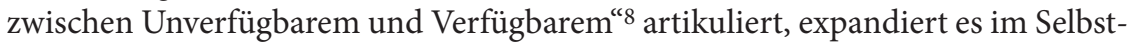
verständnis der europäischen Neuzeit, „indem Kontingenz jetzt nicht nur den Handlungen zugrundeliegt, sondern auch die Wirklichkeit erfaßt, aus der heraus

3 Schnell Alexander, Wirklichkeitsbilder, Tübingen, Mohr Siebeck, 2015, S. 82.

4 Marx Wolfgang, „Aufgabe und Struktur von theoretischer Philosophie im Rahmen des transzendental-kritischen Idealismus“, in: Marx, Wolfgang (Hrsg.), Zur Selbstbegründung der Philosophie seit Kant, Frankfurt a.M., Klostermann, 1987, S. 101.

5 Ibid., S. 102.

6 Richir M., Phänomenologische Meditationen..., op. cit., S. 402.

7 Makropoulos Michael, „Blumenberg und die Ontologie des ästhetischen Gegenstands“, in: Heidgen, Michael, Koch, Mathias und Köhler, Christian (Hrsg.), Permanentes Provisorium. Hans Blumenbergs Umwege. München, Fink, 2015, S. 95.

8 Makropoulos M., „Modernität als Kontingenzkultur. Konturen eines Konzepts“, in: v. Gravenitz, Gerhart und Marquard, Odo (Hrgs.), Kontingenz. München, Fink, Poetik und Hermeneutik 17, 1998, S. 65. 
gehandelt werden kann $[\ldots]^{\text {“99 }}$. Dergestalt entwickelt sich ein Kontingenzbewusstsein, „das nicht nur graduell, sondern prinzipiell über die traditionellen ontologischen und sozialen Beschränkungen menschlichen Handelns hinausweist " 10 . Hans Blumenberg bringt dieses Epochencharakteristikum in die prägnante wie lapidare Formel, dass die Neuzeit von dem Grundgedanken geprägt ist, „daß nicht sein muß, was ist"11.

Dies lässt auch den Begriff der Wirklichkeit nicht unberührt: Die Neuzeit, so Blumenberg an anderer Stelle, ist „nicht mehr die Epoche eines homogenen Wirklichkeitsbegriffes“12. Jedes „Realitätsbewußtsein“ vollzieht sich nun ,in der Auseinandersetzung mit einer anderen schon formierten oder sich formierenden Möglichkeit, von Wirklichkeit betroffen zu werden [... “13. Im Einklang mit diesen Beobachtungen konstatiert Bernhard Waldenfels, dass wir solche Formen der Ordnung als modern bezeichnen können, die sich aus dem „Verdacht“ heraus entwickeln, dass „die so unverbrüchlich und allumfassend scheinende Ordnung [...] nur eine unter möglichen anderen " 14 sei. Solche Formen der Ordnung sind nicht „ein für allemal gegeben, sondern unter bestimmten Bedingungen entstanden“15; sie sind nicht in maximal modal robustem Sinne notwendig, die ihnen eigenen Notwendigkeiten verweisen auf einen „Spielraum offener Möglichkeiten“16, in dessen Rahmen sie sich nicht hätten realisieren müssen. Anders formuliert: Das Kontingente als Inbegriff aller Strukturen, Ereignisse, Prozesse und Gegenstände ohne „notwendige“ Notwendigkeit, wird dadurch „zu einem konstitutiven Moment des neuzeitlichen Selbst- und Weltverständnisses“, dass es sich eben „nicht mehr auf empirische Varianten beschränkt, sondern das Ordnungsgefüge selbst antastet und vervielfältigt ${ }^{\text {"17 }}$.

Bekanntlich ist es Hume, der Kant dazu motiviert, Kontingenz als empirische „Ereignislücke“18 - die Tatsache, dass etwas geschieht, und nicht vielmehr alles so

9 Ibid., S. 65.

10 Makropoulos M, Blumenberg und..., op. cit., S. 98.

11 Blumenberg Hans, Die Sorge geht über den Fluß, Frankfurt a.M. Suhrkamp, 1987, S. 57.

12 Blumenberg Hans, „Wirklichkeitsbegriff und Möglichkeit des Romans“, in: Jauß, H.R. (Hrgs.), Nachahmung und Illusion, München, Fink, Poetik und Hermeneutik I, 1964, S. 14.

13 Ibid., S. 14.

14 Waldenfels Bernhard, „Ordnung im Potentialis“, in: Waldenfels, Bernhard, Der Stachel des Fremden, Frankfurt a.M., Suhrkamp, 1990, S. 18.

15 Waldenfels Bernhardt: „Das Geregelte und das Ungebärdige“, in: Waldenfels, Bernhard, In den Netzen der Lebenswelt, Frankfurt a.M., Surkamp, 1985, S. 82.

16 Bubner Rüdiger, Geschichtsprozesse und Handlungsnormen, Frankfurt a.M., Surhkamp, 1984, S. 38.

17 Waldenfels, B., Ordnung im Potentialis, op. cit., S. 17.

18 Espinet David, Ereigniskritik. Zu einer Grundfigur der Moderne bei Kant, Berlin, De Gruyter, Deutsche Zeitschrift für Philosophie. Sonderband 39, 2017, S. 55. 
bleibt, wie es ist -, in die Architektonik der reinen Vernunft zu integrieren. Weniger geläufig, und sicherlich weitaus kontroverser, ist die jüngst vorgelegte Interpretation von Catherine Malabou, die versucht, Kant am Leitfaden des Begriffs „Epigenese“ eine spezifische Form transzendentaler Kontingenz beizulegen.

\section{Kant (1): Kontingenz als empirische „Ereignislücke“}

Kant bescheinigt Hume, das Kausalitäts-Problem der „Verbindung von Ursache und Wirkung" so zur Darstellung gebracht zu haben, dass widerspruchslos anzuerkennen ist, „daß es der Vernunft gänzlich unmöglich sei, a priori und aus Begriffen eine solche Verbindung zu denken “19. So besteht keine Möglichkeit, „abzusehen, wie darum, weil Etwas ist, etwas anderes notwendigerweise auch sein müsse, und wie sich also der Begriff von einer solchen Verknüpfung a priori einführen lasse“20. In den Worten David Espinets: „aus der Existenz eines konkreten Gegenstandes oder Ereignisses A folgt keineswegs a priori die Existenz eines anderen konkreten Gegenstandes oder Ereignisses B“21. Die Pointe dieses „Kontingenzarguments“ ist die Unfähigkeit der Vernunft, „konkrete empirische Ereignisse zu antizipieren"22. Der Rekurs auf empirische Allgemeinheit hilft nicht weiter. Empirische Allgemeinheit ist für Kant immer nur relativ gültig, also allgemein und notwendig nur in einem Kontext, über den „spezifisches Erfahrungswissen

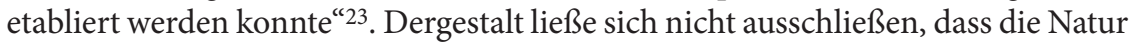
eine "so unendliche wie unverbundene Multiplizität aus Einzelereignissen“ 24 sein könnte, über die nichts Gültiges zu sagen wäre, als dass sie sich „a posteriori eben so verhalten “25. Wie vermittelt Kant diesen Befund „eine[r] rein induktive[n] probabilistische[n] Gewissheit möglicher realer Verknüpfungen"26 mit seinem Ideal apriorischer Erkenntnisgewissheit?

Sein Ansatzpunkt ist ein „minimalkausaler Modus gegenständlicher Exis-

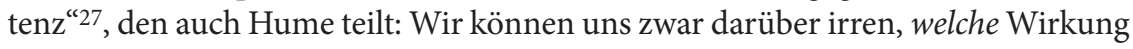
$\mathrm{X}$ das Ereignis A zur Folge hat, nicht aber dahingehend, dass Ereignis A mindestens

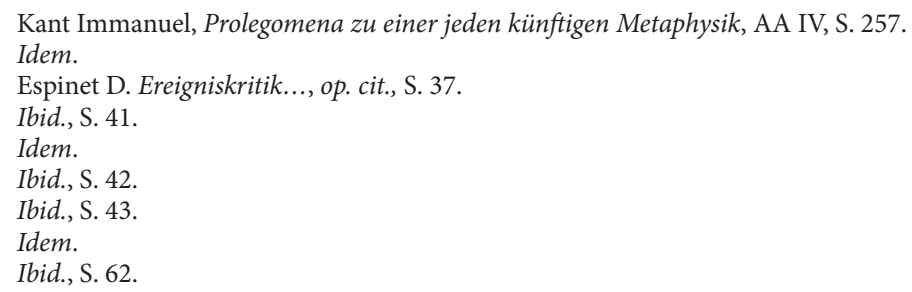


eine entdeckbarer Wirkung X zugrunde liegt - ,jede Veränderung, die man be-

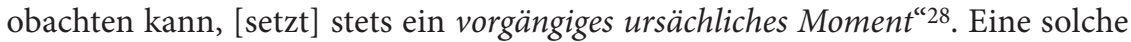
"regressive Antizipation" 29 kann nicht in Zweifel gezogen werden, lediglich progressive Antizipationen verbietet sich. Im Gegensatz zu Husserl weicht Kant damit der Möglichkeit von Ereignissen, die vollkommen neu erscheinen, „weil sie in keinerlei Erwartungshorizont vorgezeichnet “ ${ }^{30}$ waren, nicht aus. So schafft er "eine empirische Konstitutionslücke im transzendentalen Gefüge“, die a priori für „das Feld prinzipiell unantizipierbarer Wirklichkeiten ${ }^{\text {“ } 31}$ offen ist und bleibt. Denn auch wenn wir allen vergangenen Ereignissen eine „vollständige Kausaldetermination“ zuschreiben können, bleibt bestehen, dass „zu jedem Zeitpunkt potentiell auch andere Kausalverläufe möglich sind“32. Er transponiert die radikale Kontingenz Humes, welche auf eine Auflösung der Objektivierbarkeit kausaler Verhältnisse drängt, in eine „depotenzierte Kontingenz ${ }^{\prime 33}$, mithilfe derer die a priori unantizipierbare Bestimmtheit empirischer - und das bedeutet immer auch sinnlicher und zeitlicher - Gegebenheit „transzendental einzugrenzen“34.

Hierzu entwickelt er die Idee eines eingeschränkten, nur regulativen Vorgriffs: Zwar entzieht sich die spezifische Bestimmtheit von empirischen Gegebenheiten, was aber erkannt werden kann, sind Verhältnisse, in denen sich bestimmbare empirische Gegebenheiten ereignen können. So legt erste Analogie der Erfahrung fest, dass sofern etwas erscheint, das Akzidens an der beharrlichen Substanz erscheint, die zweite Analogie fixiert, dass das Erscheinen durch eine formale, aber material konkretisierbare Folgeregel strukturiert ist, die dritte Analogie postuliert ein Netz allgemeiner Wechselwirkung aller Erscheinungen, die zugleich sind. Damit regulieren die Analogie der Erfahrung die "raumzeitliche Strukturierung der anschaulichen Formen und wahrnehmbaren Realitätsgrade "35. Indem Kant auf jegliche spezifizierende Antizipation verzichtet, muss er einräumen, dass die „Bedingungen a priori der Anschauung [...] in Ansehung einer möglichen Erfahrung durchaus notwendig, die des Daseins der Objekte einer möglichen empirischen Anschauung an sich nur zufällig " 36 sind. An der Ereignislücke erweist sich, dass

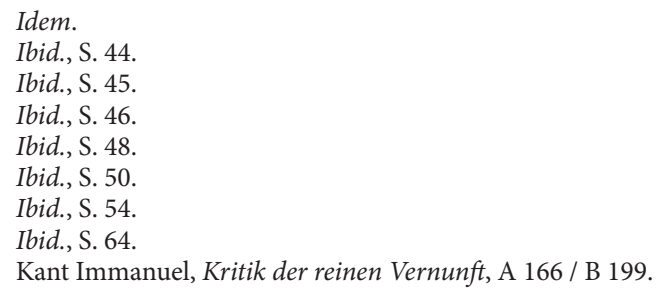




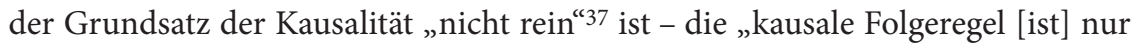
sinnvoll, wenn empirische Erfahrung tatsächlich vorliegt“" ${ }^{38}$, da „Veränderung ein Begriff ist, der nur aus der Erfahrung gezogen werden kann" ${ }^{\text {“39. }}$

Es geht nun nicht darum, die Güte des Kantischen Ansatzes zu bewerten, sondern die Konsequenzen dieser Stoßrichtung dieses Umgangs mit Kontingenz für seine transzendentale Theorie-Anlage in den Blick zu bekommen. Kant ist sichtlich bemüht, die Ereignislücke, zu der ihn Humes Kontingenzargument zwingt, „so trivial wie möglich zu halten, ohne sie jedoch vollständig zu schließen “40. Der „Kontingenzrest", ein „a priori nicht antizipierbarer Rest" 41 , der bestehen bleibt, verweist an dasjenige, in das wir nichts „hineinlegen“ können, dem wir nichts a priori vorschreiben können: „Da er nicht von uns in die Dinge hineingelegt wird, muss er in den Dingen selbst liegen - in den Dingen, wie sie unabhängig von unseren Erkenntnisbedingungen sind“ 42 . Das System der reinen Vernunft muss demnach eine „bewegliche Mitte“ zwischen Subjekt-Abhängigkeit und Subjekt-Unabhängigkeit aufweisen, die zwischen den Polen "antizipierbarer Determination“ und „unantizipierbarer Indetermination“ zu oszillieren vermag, „ohne dass doch der Zustand reiner ontologischer Verwirklichung auf der einen oder anderen Seite jeweils erreicht werden könnte oder überhaupt als realer Zielpunkt angenommen werden dürfte" 43 . Als transzendental ist die hier architektonisch berücksichtigte Kontingenz deshalb nicht zu bezeichnen, weil „die reine Apperzeption mit der ihr eigenen Art von (kategorialer) Notwendigkeit" 44 von ihr prinzipiell unberührt bleibt, und somit das transzendentale Ordnungsgefüge in seiner apriorischen inneren Notwendigkeit nicht „selbst antastet und vervielfältigt “ ${ }^{45}$. Anders formuliert: Trotz einer signifikanten Integration von Kontingenz in die Architektonik der reinen Vernunft findet keine Erweiterung der generativen Matrix des „transzendentalen Erfahrungsnetzes" 46 statt.

\footnotetext{
Ibid., B 3.

Espinet D. Ereigniskritik..., op. cit., S. 66.

Kant I., Kritik der reinen Vernunft, B 3.

Espinet, D. Ereigniskritik..., op. cit., S. 55.

1 Willaschek Marcus, „Affektion und Kontingenz“, in: Schumacher, R. (Hrgs.), Idealismus als Theorie der Repräsentation, Paderborn, Mentis, 2001, S. 229.

42 Ibid., S. 229.

43 Espinet D. Ereigniskritik..., op. cit., S. 19.

44 Hoppe Hansgeorg, Synthesis bei Kant. Das Problem der Verbindung von Vorstellungen und ihrer Gegenstandsbeziehung in der Kritik der reinen Vernunft. Berlin, De Gruyter, 1983, S. 110.

45 Waldenfels, B., Ordnung im Potentialis, op. cit., S. 17.

46 Espinet D. Ereigniskritik..., op. cit., S. 63.
} 


\section{Kant (2): Kontingenz als „transzendentale Epigenese“}

Wie gemeinhin bekannt ist, möchte Kant in der Transzendentalen Deduktion einen Beweis des apriorischen Bezugs der Kategorien auf Gegenstände erbringen. Im $₫ 27$ befasst er sich mit dem Problem, wie eine notwendige Übereinstimmung zwischen den Kategorien und den Gegenständen der Erfahrung zu konzeptualisieren ist. Im Fokus steht die Abhängigkeit von zwei grundlegenden Strukturen: dem a priori Ursprung der reinen Begriffe des Verstandes und dem a priori Ursprung der Beziehung dieser Begriffe zu den Gegenständen der Erfahrung. Kant schreibt:

Nun sind nur zwei Wege, auf welchen eine notwendige Übereinstimmung der Erfahrung mit den Begriffen von ihren Gegenständen gedacht werden kann: entweder die Erfahrung macht diese Begriffe, oder diese Begriffe machen die Erfahrung möglich. ${ }^{47}$

Der erste Weg, Kategorien als „empirischen Ursprungs“48 anzusetzen, verbietet sich, da Kategorien Begriffe a priori sind, und damit unabhängig von der Erfahrung. Folglich bleibt der zweite Weg, „daß nämlich die Kategorien von Seiten des Verstandes die Gründe der Möglichkeit aller Erfahrung überhaupt enthalten“49. Dieser Weg sei „gleichsam ein System der Epigenese der reinen Vernunft“50. Kant schlägt noch einen „Mittelweg“ vor, der den Ursprung der Kategorien weder in der Erfahrung, noch in einer prinzipiellen Apriorizität verortet. Dieses „Präformationssystem der reinen Vernunft “51 verstünde die Kategorien ,als uns mit unserer Existenz zugleich eingepflanzte Anlagen zum Denken“52. Dergestalt käme der Geltungsanspruch der Kategorien nie über eine „subjektive Notwendigkeit“53 hinaus, wodurch ihnen „die Notwendigkeit mangeln würde, die ihrem Begriff wesentlich angehört" 54 - eine „objektive Notwendigkeit [...], welche die reinen Verstandesbegriffe (und die Grundsätze ihrer Anwendung auf Erscheinungen) charakterisiert $[\ldots]^{\text {“ } 55}$.

Wie aber kann mithilfe des Begriffs einer Epigenesis der reinen Vernunft die apriorische Gültigkeit gegenständlicher Bezugnahme gerechtfertigt werden?

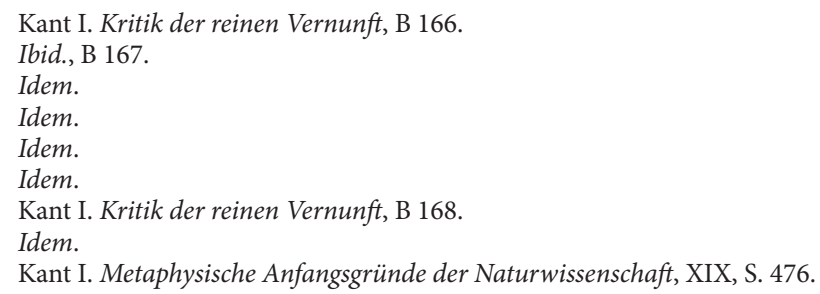


Allgemein bezeichnet „Epigenese“ den produktiven Prozess einer (Selbst-)Differenzierung und (Selbst-)Komplexifizierung. Malabou zufolge artikuliert Kant mit diesem Begriff folgende Einsicht: „The agreement between the categories and objects can only be thought as the product of a dynamic, creative, and self-forming relation" ${ }^{56}$. Die eigentliche Aufgabe der Transzendentalen Deduktion wäre somit, eine "coincidence between differential growth and the a priori“ aufzuzeigen, die in ihrer Interpretation „a founding transformability in the transcendental“ ${ }^{157}$ andeutet. Zur Disposition steht also derjenige Prozess, in dem sich das „Transzendentale“ als Bedingung der Möglichkeit von Erfahrung und Erkenntnis „selbst formt“: „[h] $\left[\right.$ ow it constitutes itself as the condition of the forms of thought ${ }^{458}$. Die Formulierung, wonach der Verstand die Gründe der Möglichkeit aller Erfahrung "enthalte", sei demnach nicht so verstehen, dass diese Gründe in ihm schon fertig und unveränderlich vorliegen, sondern als Potentiale fungieren, die einer spezifischen Entwicklung bedürfen, um das phänomenale Profil der Gegenstände der Erfahrung zu ermöglichen. Soll heißen: Die Spontanität des Verstandes appliziert eine Form auf das Gegebene, und erwirbt dabei Erkenntnis als Produkt seiner eigenen Aktivität, wobei diese Erkenntnis retroaktiv in die Ermöglichung seiner Applikation einfließt. Die gegenstands- und erfahrungskonstitutiven Regeln basaler Kategorialität etablieren so ,ein wechselseitiges Bedingungsverhältnis mit dem Konstituierten "59, das imstande ist, diese Regeln wiederum zu modifizieren.

Zwei skeptische Optionen drängen sich auf: Wie die Rolle der Erfahrung nicht dahingehend schmälern, dass die Epigenesis sich doch auf eine Form der Präformation reduzieren lässt, die Möglichkeit der Übereinstimmung also schon auf spezifische Potentialitäten des Transzendentalen rückgeführt, und nicht erst in einem transformativen Prozess wechselseitiger Ko-Konstitution von Erfahrungsbedingungen und Erfahrung generiert wird? Wenn die Rolle der Erfahrung affirmiert wird, wie diese nicht auf eine Weise überziehen, welche die Erfahrungsbedingungen als autonomen oder „reinen“ Geltungsbereich auflöst? Es würde den Rahmen dieser Überlegungen sprengen, hierauf Antworten anzuführen. Allgemein kann festgehalten werden: Wenn der Begriff der Epigenese genutzt werden soll, um eine Entwicklung des Transzendentalen - genauer: eine „specific determination of the generative relation between a priori and a posterior " $" 60$ - zu denken, verhindern präformationistische, dogmatisch-idealistische und empiristische Definitionen,

\footnotetext{
Malabou Catherine, Before Tomorrow. Epigenesis and Rationality, Malden, Polity, 2016, S. 25.

Ibid., S. 18.

Ibid., S. 4.

Schnell A. Wirklichkeitsbilder, op. cit., S. 108.

Malabou C. Before Tomorrow..., op. cit., S. 52.
} 
die Notwendigkeit der Kategorien ebenso wie die Notwendigkeit der Beziehung zwischen Kategorien und Gegenständen zu rechtfertigen. Malabou konstatiert unmissverständlich wie sachlich richtig: „If the unity of nature is necessary, it is because the agreement of categories with objects is produced spontaneously and depends on nothing other than itself" 61 .

Der philosophische Ertrag einer epigenetischen Perspektive offenbart sich demnach gerade nicht, wenn diese Spontaneität im Sinne einer Rückführbarkeit-auf... vorgestellt wird. Vielmehr gilt es, die Frage nach der unerschütterlichen inneren Natur des Verstandes, im Sinne einer „Wurzel“, eines „Zentrums“, oder eines invariablen „Fokus“ der Notwendigkeit von Kategorien und deren Übereinstimmung mit Gegenständen, durch die Frage zu ersetzen, wie sich „Punkte des Kontakts“ zwischen a priori und a posteriori bestimmen und architektonisch lokalisieren lassen. Das bedeutet:

[T] he questions about the stability of the transcendental should not be questions that [...] seek to determine the innate nature of the cognitive power or, conversely, its mysterious makeup. Stability cannot be traced back to the focus. In Kant the transcendental is a surface structure. ${ }^{62}$

Transzendentale Genesis wäre demnach ein Prozess der Kontaktsuche der kreativen wie limitierten Ressourcen des Transzendentalen mit den Möglichkeiten seines eigenen „Außen“. Diese Kontaktsuche gleicht einer Improvisation, die Ordnung erzeugt, eine Improvisation, die spezifisch orientiert ist, ohne jemals völlig bestimmt zu sein. Als „Kontaktpunkte“ erweisen sich erfolgreich stabilisierte Selbstdifferenzierung von Strukturen zwischen Erfahrungsbedingungen und Erfahrung: „The dynamic of transcendental philosophy proceeds both from the formal anteriority of the a priori - the archeological dimension - and from its modifiability through successive correction - the teleological dimension"63. Wie in Espinets Interpretation der architektonischen Konsequenzen von Humes Kontingenzargument kommt auch hier die Figur einer „mobilen Mitte“ ins Spiel, die den eigentlichen „Kern“ der Vernunft bildet, und nun nicht zwischen antizipierbarer Determination und unantizipierbarer Indetermination, sondern zwischen archäologischer Fundierung von schon entdeckter „Form“ und teleologischer Ausrichtung auf noch nicht entdeckte „Form“ oszilliert.

$61 \quad$ Ibid., S. 70.

62 Ibid., S. 36.

63 Ibid., S. 174. 
Von entscheidender Bedeutung ist, dass weder eine Totalisierung der archäologischen Momente als Grund, noch eine Totalisierung der teleologischen Momente als Ziel, die Notwendigkeit der Kategorien und ihrer Übereinstimmung mit Gegenständen letztgültig fundieren kann: „The transcendental is subject to epigenesis - not to foundation“64. „Epigenesis des Systems der reinen Vernunft“ hieße dann: Wir bleiben verwiesen an eine Spontaneität, die sich als dissipatives Element und damit als das schlechthin Irreduzible der Apriorizität erweist. Sie ist gerade nicht in den „Tiefen" einer Wurzel, der "Sicherheit" eines Grundes, oder der "Gewissheit“ eines Zieles zu verorten, sondern „empfängt“ sich stets an oberflächlichen Kontaktpunkten, die „nicht hierarchisch, sondern in unterschiedlichsten Beziehungsgeflechten organisiert sind“" 65 . Malabou betont: „The spontaneity of the understanding cannot reach itself substantially, and it is precisely due to this that it is impossible to think it as predetermined or given"66. Demzufolge ist jede Kategorie, insofern sie notwendig sein soll, genuin entdeckte, weil spontan synthetisierte - und eben nicht prädeterminierte oder gegebene - Kategorie, und jede Übereinstimmung mit Gegenständen, insofern sie notwendig sein soll, genuin entdeckte, weil spontan synthetisierte - und eben nicht prädeterminierte oder gegebene - Übereinstimmung. So ist auch das System der Kategorien kontingent, ohne „dass damit gesagt wäre, dass die Elemente und die Struktur des Systems in bezug auf dieses selbst kontingent sind“67. Die „innere“ Notwendigkeit des Zusammenspiels von Elementen und Struktur des Systems lässt sich erst begründen, wenn die „spezifisch menschliche Erkenntnisform“68, die sich zufälligerweise ausgebildet hat, ihrer selbst hinreichend gewahr wird. Anders formuliert: Die Rede von einer Notwendigkeit des Systems der Kategorien ist transzendentalphilosophisch erst dann sinnvoll, wenn ebendiese Erkenntnisform als spezifizierbare architektonische Einheit entdeckt ist - ohne Entdeckung ,innerer“ Einheit keine Begründbarkeit „innerer“ Notwendigkeit.

Daran anknüpfend noch einige Bemerkungen zu den modaltheoretischen Konsequenzen der Verweigerung klassischer Figuren der Letztbegründung, welche im Begriff der Epigenesis operativ wirken. Spätestens mit der Kritik der Urteilskraft wird eine Neu-Akzentuierung von „Kontingenz" und „Notwendigkeit“ virulent, die in der Kritik der reinen Vernunft im Vorhaben einer theoretischen

64 Ibid., S. 158.

65 Poser Hans, „Wissenschaftsmodelle des Neuen und ihre Grenzen. Kreativität und die Theorien der Komplexität“, in: Abel, Günther (Hrgs.) Kreativität, Hamburg, Meiner, 2006, S. 972.

66 Malabou, C. Before Tomorrow..., op. cit., S. 98.

67 Bunte Martin, Erkenntnis und Funktion. Zur Vollständigkeit der Urteilstafel und Einheit des kantischen Systems, Berlin, De Gruyter, 2016, S. 30, Fn. 85.

68

Ibid., S. 30. 
Selbstbestimmung der reinen Vernunft noch hinreichend domestiziert scheint. Zentral ist die Neubestimmung der Stelle in der Architektonik der Vernunft, von der aus sich die Intelligibilität der Frage nach der Unterscheidung zwischen Kontingenz und Notwendigkeit bemisst. Kant wichtigste Pointe betrifft das Kontingente nicht als Möglichkeit radikaler Alterität zum Notwendigen, sondern als die Möglichkeit verschiedener Register des Notwendigen. „Kontingent“ bezeichnet damit in erster Linie nicht die möglichen Alternativen zu den Notwendigkeiten der geordneten Strukturen der Natur, sondern die möglichen Unabhängigkeiten von geordneten Strukturen und dem System der Vernunft. Kontingent sind diejenigen Ordnungen, die auch ohne subjekthafte Spontaneität notwendig sein können: Eine Fülle morphogenetischer Selbststabilisierungen von geordneten Strukturen in einer wechselnden Umwelt, die kraft ihrer architektonischen Eigenkomplexität die Frage nach ihrer „inneren“ Notwendigkeit aufwerfen. In seinem vielfach zitierten Beispiel schreibt Kant:

Es mag immer sein, daß z.B. in einem tierischen Körper manche Teile als Konkretionen nach bloß mechanischen Gesetzen begriffen werden könnten (als Häute, Knochen, Haare). Doch muß die Ursache, welche die dazu schickliche Materie herbeischafft, diese so modifiziert, formt und an ihren gehörigen Stellen absetzt, immer teleologisch beurteilt werden, so daß alles in ihm als organisiert betrachtet werden muß, und alles auch in gewisser Beziehung auf das Ding selbst wiederum Organ ist. ${ }^{69}$

Zunächst gilt hier das Gleiche wie für das System der Kategorien: Der Kategorienapparat mag im Ganzen zufällig sein, der Zusammenhang seiner Elemente und Strukturen ist es nicht; der tierische Körper mag im Ganzen zufällig sein, der Zusammenhang seiner Elemente und Strukturen ist es nicht. Während die traditionelle Gegenüberstellung von Kontingenz und Notwendigkeit als dasjenige, was auch anders oder nicht möglich ist, und dasjenige, was nicht anders sein kann, im Rahmen mechanischer Kausalität plausibel bleibt, trifft dies für die Selbstorganisation von Struktur nicht länger zu. Das Notwendige als das, was unmöglich nicht sein kann, verfehlt die innere Notwendigkeit des Schönen, verfehlt die innere Notwendigkeit des tierischen Körpers, verfehlt die innere Notwendigkeit des Kategorienapparats. Diese Notwendigkeiten können ohne jeden Widerspruch nicht sein - sie haben kein Gegenteil. Ihre Bestimmungen sind Ausdruck transzendentaler Kontingenz. „Transzendental kontingent“ ist all das, was „nach unserer Verstandeseinheit zufällig sein [möge]“, dennoch „aus einem, wenn gleich uns

69 Kant I., Kritik der Urteilskraft, B 298. 
unbekannten, Prinzip der Einheit des Mannigfaltigen als notwendig angesehen werden müssen “70. „Transzendentale Epigenesis“ verweist dementsprechend auf den Prozess einer weder empirisch, noch ontologisch, noch metaphysisch gänzlich zu fundierenden allgemeinen Morphogenese. Im Anschluss daran indiziert „Transzendentale Epigenesis des Systems der reinen Vernunft“ denjenigen Prozess, der die inneren Notwendigkeiten des Systems der reinen Vernunft bestimmt, um offenzulegen, „unter welchen Bedingungen die Strukturen unserer Zuwendung genau so ,real' oder ,objektiv' sein können"71 wie dasjenige, dem wir uns zuwenden. Damit ist sich die reine Vernunft ihr erster Gegenstand: Inbegriff einer „anderen“ Notwendigkeit, die transzendental kontingent ist in dem Maße, in welchem sie registriert, dass sie selbst „the existence of differerent levels of necessity or lawfulness"72 in Anspruch nehmen muss, um die Idee ihrer eigenen Einheit zu bilden. So kann „unsere Erkenntnis über eine Einheit verfügen [...], die rein architektonisch zu begründen ist" "73. Architektonik wird letztlich sichtbar als transzendentales „Prinzip der Suche“ des Systems der reinen Vernunft nach sich selbst. Transzendental ist dieses Prinzip deshalb, weil die Faktizität heterogener Notwendigkeitsregister sich als Bedingung seiner eigenen Möglichkeit erweisen - es ist nicht gründbar. Im Wesentlichen besteht es „aus einer richtungsorientierenden Reflexion"74, weshalb Kant Architektonik auch als „zetetisches Verfahren“ einer „critischen metapyhsik“75 fasst. Dieses grenzt er vom „dogmatischen Verfahren“ einer „doctrinären metaphysik“ streng ab, das stets der Illusion verfällt, bereits oder immer schon über den Inbegriff aller Ideen - also aller architektonisch konfigurierbaren Einheiten möglicher Erkenntnis - zu verfügen.

\section{Richir: Kontingenz als Spur der "Sache selbst ${ }^{\prime \prime}$}

Vor dem Hintergrund dieser durchaus kontroversen Interpretationen von Espinet und Malabou lässt sich Richirs direkter Anschluss an die kantische Fragestellung als Transformation des Motivs transzendentaler Kontingenz formulieren:

$70 \quad$ Ibid., S. 180.

71 Gabriel Markus, „Ist die Kehre ein realistischer Entwurf?“, in: Espinet, David und Hildebrandt, Toni (Hrgs.), Suchen, Entwerfen, Stiften. Randgänge zum Entwurfsdenken Martin Heideggers. Paderborn, Fink, 2014, S. 90.

72 Malabou, C. Before Tomorrow...., op. cit., S. 173.

73 Rivero Gabriel, Zur Bedeutung des Begriffs Ontologie bei Kant. Eine entwicklungsgeschichtliche Untersuchung, Berlin, De Gruyter, 2014, S. 21.

74 Ibid., S. 38.

75 Kant I., „Reflexion 4455“, AA XVII, S. 558. 
Alle Register der Notwendigkeit werden im Rahmen seiner „Mathesis der Instabilität" hyperbolisch aufgebrochen, um im Gegenzug zu einer Differenzierung dieser Register in fundierende und fundierte Register zu gelangen; eine Differenzierung, die immer wieder neu in der Auseinandersetzung mit konkreten phänomenalen Gehalten zu erfolgen hat. „Register“ bezeichnet dabei ein „Konnex derjenigen Strukturelemente“, die ein je spezifisches „Feld von Möglichkeiten“" 76 am Leitfaden spezifischer innerer Notwendigkeiten konstituieren. Nehmen wir die „kategoriale Anschauung" Husserls als Beispiel: Die "phänomenologische Basis“ von dieser bildet „der intentionale Sinn [...] eines beliebigen anschaulich dargestellten Gegenstandes“, so dass „diese oder jene Darstellung von Gegenständen vorab

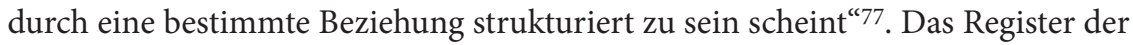
kategorialen Anschauung etabliert sich als ob die phänomenologische Basis „niemals da gewesen wäre außer immer schon durch all die Beziehungen strukturiert, welche ihrerseits im fundierten Register möglich sind“, also eben im Register der kategorialen Anschauung. Die „Verformung“, die im Übergang vom fundierenden Register zum fundierenden Register stattfindet, wirkt also, „als ob diese Grundlagen selbst immer schon das fundierende Register für das fundierte Register wäre“. Doch die phänomenologische Basis des fundierenden Registers ist im fundierten Register gerade nicht „vernichtet“, sondern nur „ausgestrichen“, oder wie Richir

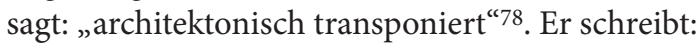

Das phänomenologische Feld kann derart als ein Stapel architektonischer Register in den Blick genommen werden, die wechselweise als fundiertes Register, phänomenologische Grundlage und fundierendes Register für ein für ein weiteres fundiertes Register fundieren können, womit einhergeht, was ganz entscheidend ist, dass dieser Funktionswechsel nicht Akten des Bewusstseins unterstehen, die in irgendeiner Gegenwart der so genannten ursprünglichen Zeit angewiesen werden können. ${ }^{79}$

Zwischen irreduziblen Registern eröffnen „sich jedes Mal in jeder Bestimmtheit [...] Unbestimmtheitszonen, die unaufhörlich in einem hiatus, schweben'“80. Diese „Unbestimmtheitszonen“ erstrecken sich „,on der einfachsten Wahrnehmung

\footnotetext{
6 Flock Philip, Das Phänomenologische und das Symbolische. Marc Richirs Phänomenologie der Sinnbildung in Auseinandersetzung mit dem symbolischen Denken, Dissertation Wuppertal, 2018, S. 44.

77 Richir M., „Über die phänomenologische Revolution. Einige Skizzen“, in: Gondek, Hans-Dieter, Klass, Tobias und Tengelyi, László, Phänomenologie der Sinnereignisse, München, Fink, 2011, S. 71.

78 Ibid., S. 72.

79 Ibid., S. 72 f.

80 Ibid., S. 75.
} 
bis zu den subtilsten logischen Formen “81. Dies hat unweigerlich zur Folge, dass sich Architektonik als zetetisches Verfahren radikalisiert: Zetetisch arbeitet Richir zufolge eine Philosophie dann,

wenn es ihr gelingt, mit Unbestimmtheiten zu, arbeiten', die nicht im Chaos schweben, sondern miteinander verknüpft sind [...] durch ihre wechselseitige dynamische Situierung, in der ihre jeweilige Beweglichkeit ins Spiel kommt. ${ }^{82}$

Konkretisieren wir dies: Humes Kontingenzargument führt zu einer „beweglichen Mitte“ im System der einen Vernunft, zwischen „antizipierbarer Determination" und „unantizipierbarer Indetermination“; der Gedanke einer transzendentalen Epigenese veranschlagt ebenfalls eine „bewegliche Mitte“, und zwar zwischen „Archäologie“ und „Teleologie“. Hier zeigen sich vier basale Parameter einer $z e$ tetischen Matrix transzendentaler Architektonik: Jede architektonische Konfiguration muss, insofern sie architektonische Konfiguration einer Einheit möglicher Erkenntnis und nicht nur deren gegenständliche (Re-)Identifikation sein will, die Nicht-Totalisierbarkeit dieser Parameter implementieren. Im Spannungsfeld der Unmöglichkeit totaler antizipierbarer Determination, der Unmöglichkeit totaler unantizipierbarer Indetermination, der Unmöglichkeit totaler archäologischer Gründung, und der Unmöglichkeit totaler teleologischer Finalisierung formiert sich die Möglichkeit einer Architektonik, „die zumindest dem Anspruch nach jede metaphysische Entscheidung ausschließt, insofern sie eine Architektonik von Fragen und Problemen sein und bleiben muß, die in Wirklichkeit unendlich ist" ${ }^{\text {"83. }}$

Diese Re-Akzentuierung des Architektonik-Begriffs vermag dem Umstand Rechnung zu tragen, dass die "Sache selbst“ nie etwas ist, „das wir vorweg anschauen könnten“. Um epistemischen Kontakt mit ihr herzustellen und zu wahren, muss sie „als solche offengehalten“ werden, was impliziert, von allen „verfrühten Antworten“ abzusehen, die sich „in ihrer scheinbaren Positivität einschleichen wollen ${ }^{\text {" }}{ }^{4}$. Architektonik fungiert dabei als dasjenige Instrument transzendentaler Methodenlehre, das erlaubt, „der transzendentalen Illusion eines anschaulichen Verstehens ${ }^{\text {“85 }}$ nicht nachzugeben. Richir schreibt:

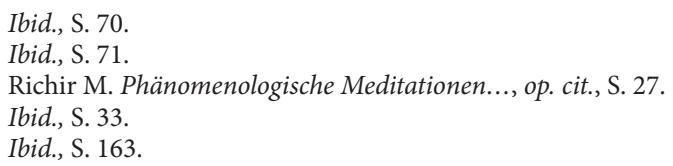


Die ganze Schwierigkeit dieser Reflexion liegt darin, daß sie jede metaphysische oderontologische Bestimmung des »Seins« des Phänomens ausklammert und damit das transzendentale Feld der Phänomene als ein architektonisches eröffnet, nämlich das noch phänomenologisch zu erarbeitende Feld der Begegnung zwischen unserem Denken und der radikalen [...] Kontingenz der Phänomene in ihrer Phänomenalisierung. ${ }^{86}$

Hier erreichen wir den „tiefsten Punkt“ transzendentaler Theorie-Anlage: Jede Präfiguration, „welche Formen des Wissens und welche Formen des Erschei-

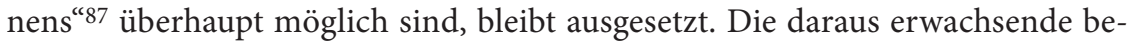
gründungstheoretische Aufgabe ist klar: Wie können sich Register innerer Notwendigkeit angesichts einer solchen Kontingenz ausbilden und ko-existieren?

Die Methode, die uns Richir zufolge auf den „Weg“ bringen kann, diese $\mathrm{Zu}$ sammenhänge auf systematische Weise phänomenologisch zu untersuchen, ist die „hyperbolische Epoché“. Genau das ist dementsprechend das methodische Profil, das Richir der hyperbolischen Epoché zuschreibt: Sie „,erweckt' [...] sozusagen das Flimmern des Scheins in den Phänomenen" ${ }^{\text {"88. }}$. Vergegenwärtigen wir uns das mit einer längeren Passage von Richir:

Der Schein erscheint (wenn man sich metaphorisch auf das Flimmern eines Sterns beziehen mag) wie eine Art Licht ohne existierenden Träger; aber andererseits und dazu korrelativ ist der Schein nach Maßgabe dieser Instabilität immer im Begriff, von der einen oder anderen intentionalen Struktur eingenommen und dadurch in Erscheinungen zerschnitten zu werden, die intuitiv diesen oder jenen bereits gestifteten intentionalen Sinn erfüllen [...]. Geht man also zum Status der Erscheinungen über, [...] scheint das ,Licht' der Schein-Erscheinungen durch eine existierende Quelle ausgesandt zu sein - die Quelle der Stiftung, in der sie ihren Sinn finden. Allerdings scheint diese Bewegung unter dem Ordnungssystem der hyperbolischen Epoché ebenso instabil wie die erste zu sein, weswegen es sich gerade um ein Flimmern zwischen zwei Extrempolen handelt, das in seinem Zentrum von dem unbeherrschbaren Umschlag von einem Pol zum anderen bewohnt wird [...]. ${ }^{89}$

Die Epoché hyperbolisch zu verstehen bedeutet demnach, den Schein durch hyperbolisch betriebene „architektonische Umwälzung "90 von seinen in-

\footnotetext{
Ibid., S. 57. Hervorhebung F.E.

Gabriel Markus, Sinn und Existenz. Eine realistische Ontologie, Berlin, Suhrkamp, 2016, S. 464.

88 Richir M, „Epoché, Flimmern und Reduktion in der Phänomenologie“. in: Bernet, Rudolf und Kapust, Antje (Hrgs.), Die Sichtbarkeit des Unsichtbaren, München, Fink, 2009, S. 37.

89 Ibid., 37.

90 Idem.
} 
tentionalen Vereinnahmungen zu befreien, um ihn in seinen grundlegenden Unbestimmtheiten indirekt, im Rücken der gegenständlichen Struktur unserer Erfahrung, aber dennoch in „undarstellbarer Konkretheit", nicht-inferentiell registrierbar zu machen, und ihn in seinen Zusammenhängen thematisch werden zu lassen. Versuchen wir „[d]iese Situation, auf die sich die hyperbolische phänomenologische Epoché öffnet" ${ }^{\text {"91 }}$, noch etwas genauer zu betrachten. Das besagte "phänomenologische Flimmern" weist eine zweifache Richtung auf: Der Schein flimmert in den „gegenständlichen Aspekten“ unserer Erfahrung auf seinen „Erscheinungsstatus" hin, also auf sein Eingebunden-Sein in intentionale Strukturen verschiedener doxischer Modalitäten, und er flimmert in den „Entzugsmomenten“ unserer Erfahrung auf seinen „Scheinstatus“ hin. In jeder Re-Kontingenzierung („Aufhebung“) und Re-Aktualisierung („Setzung“) des Gegenstandes einer intentionalen Bezugnahme sorgen die „,Spiele‘ der augenblicklichen Umschlagsbewegungen der flüchtigen Versammlungen und Zerstreuungen des Scheins" zwischen dem Erscheinungsstatus und dem Scheinstatus dafür, dass wir weder mit unserer Erfahrung als Ganze „verkleben“, noch dass wir „Bruchkanten“ gegenwärtigen müssten, weil etwas in seinem Erscheinungsstatus "gesetzt" wurde, ohne auf seinen Scheinstatus hin durchlässig geblieben - und das heisst hier „aufhebbar" - zu sein. Um dies zu verdeutlichen, verweist Richir auf die Erfahrungsmodi der Phantasie und des Traumes, die in seiner Neugründung der Phänomenologie eine zentrale, man könnte sagen "paradigmatische“ Rolle einnehmen. So schreibt er, dass

etwas von diesen unbeherrschbaren und unverhofften ,Spielen' der Scheinmodi untereinander [...] irgendwo im Hintergrund der scheinbar ungezügelten Spiele der Phantasie und des Traums [schwebt], das heißt der ,Assoziationen', die dort im Einfall von jeder intentionalen Ausrichtung frei zu sein scheinen. ${ }^{93}$

Diese „architektonischen Ebenen“, die hier in den Blick geraten, unterhalten keinerlei „teleologische“ Beziehung zu den Registern, die auf verschiedene Weise an ihnen als "phänomenologischer Nahrung" partizipieren; dies tun sie ohne sie sich ganz einzuverleiben, aber nicht ohne ihnen eine spezifische Richtung „vorzugeben“, eben auf imaginäre, ideale, erinnerte oder wahrgenommene Gegenständlichkeit. Dementsprechend führt Richir weiter aus:

\footnotetext{
Ibid., S. 36.

Ibid., S. 38.

Ibid., S. 41
} 
Man versteht, dass der Übergang von der architektonischen Ebene des Scheins [...] zur architektonischen Ebene der Erscheinungen (die durch einen Typ der Stiftung zum intentionalen Sinn in Beziehung gesetzt sind) eine ,Verformung ist, die wir eine , architektonische Umgestaltung' nennen, insofern sie auch ein Übergang im Hiatus ist, wo es von einer Ebene zu einer anderen keinerlei logisch-eidetische ,Deduktion' oder mögliche inhaltliche Ableitung gibt. ${ }^{94}$

Um unser Beispiel von vorhin aufzugreifen: Die kategoriale Anschauung ist das Ergebnis einer solchen „architektonischen Umgestaltung“, in der die spezifische „phänomenologische Nahrung " zu „verschwinden“ scheint - das fundierende Register der sinnlichen Wahrnehmung -, ohne dass dabei je geltend gemacht werden könnte, das es vom fundierenden Register der sinnlichen Wahrnehmung zum fundierten Register der kategorialen Anschauung einen „notwendigen“ Übergang gibt. Diese Umgestaltung, welche die ganze Kontingenz „absorbiert“, die im Übergang von fundierendem zu fundiertem Register liegt, macht nun Richir zufolge „wie von hinten oder wie von unten den stets dunklen Teil des Sinnes“ aus, der ihm „etwas vom Lebendigen [à-vif] oder der Lebendigkeit der Phänomene verleiht" ${ }^{\text {"95 }}$. Im Bild formuliert: Es ist dieser „dunkle Teil“, durch den „Sinn“ als der „Spielraum“, in dem "das Reale in seinem minimalen - aber notwendigen - Wahrheitsbedingungen erscheint ${ }^{\text {“96 }}$, in einem spezifischen Sinne „vibriert", er esse in actu ist und bleibt. Folglich benötigen wir eine Methode, die es uns gerade nicht „erlaubt, [...] uns auf Dauer ,stabil' in ihr einzurichten “97, sondern uns immer wieder dazu anhält, „bereits in den Erscheinungen und den Erscheinenden die intentionalen Strukturen selbst auszuschalten und zu überraschen. Den „Schein hinter den Erscheinungen schweben“ zu lassen, „ohne ihn fixieren zu können“ 98 , bedeutet, in jeder Bezugnahme immer wieder den nicht einholbaren Rest in seiner „undarstellbaren Konkretheit“ thematisch zu halten, „dessen konstitutiver Abwesenheit sich die Dynamik der Strukturbildung "99 - und das heisst immer auch: der „unscheinbaren“ Architektonik der Strukturbildung - von Erscheinendem verdankt. Phänomenologisch gilt: „Seine Fixierung würde ihn unmittelbar und blind in (sinnliche,

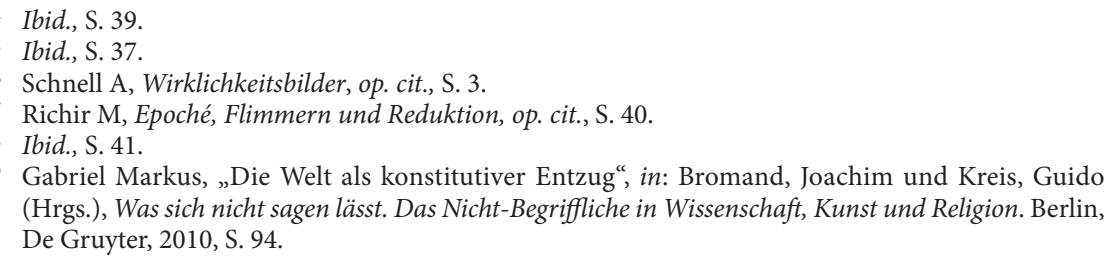


phantastische, denkbare usw.) Erscheinungen umgestalten. “100 Das Ausmaß, das eine solche Abwesenheit in der "Selbstvergessenheit" der Erfahrung anzunehmen imstande ist, sollte gerade „nicht als Mangel gewertet werden, sondern als Indiz für Komplexität "101. Kurz: Der unscheinbare Mangel, der alles gegenständlich Erscheinende „durchzieht“, ist gerade die Bedingung der Möglichkeit seiner architektonischen Komplexität. Und nichts anderes ist Phänomenalisierung: Ein „kreativer Prozeß wachsender Komplexität auf immer höherem Niveau"102. Diesen Prozess als das Real-Sein-Müssen eines zetetischen Prinzips verstehen zu lernen: Das ist unserer Meinung nach Richirs dringender und berechtigter Auftrag zur Aktualisierung der „kopernikanischen Revolution“ an die gegenwärtige Philosophie.

Fabian Erhardt, lehrt Philosophie und Allgemeine Rhetorik an der Universität Tübingen. Derzeit Promotion bei Prof. Alexander Schnell in Wuppertal. Diverse Publikationen im Themenkreis zeitgenössischer Phänomenologie.

100 Richir M, Epoché, Flimmern und Reduktion, op. cit., S. 41.

101 Hogrebe Wolfram, Wer im Mythos lebt..., in: Hogrebe, Wolfram (Hrgs.), Echo des Nichtwissens, Berlin, Akademie, 2006, S. 341.

102 Poser Hans, „Wissenschaftsmodelle des Neuen und ihre Grenzen. Kreativität und die Theorien der Komplexität“, in: Abel, Günther (Hrgs.) Kreativität, Hamburg 2006, S. 978. 